\title{
Factorial moment analyses in diffractive lepton-nucleon scattering
}

\author{
Zhang Yang \\ Institut für theoretische Physik, FU Berlin, Arnimallee 14, 14195 Berlin, Germany
}

\begin{abstract}
It is pointed out that "the colorless objects" in diffractive lepton-nucleon scattering in the small- $x_{B}$ region can be probed by measuring the scaled factorial moments of final-state-hadrons and the dependence of their scaling behavior upon the diffractive kinematic variables. The Monte Carlo implementation of RAPGAP and JETSET are discussed as illustrative examples. The results of these model-calculations show in particular that inclusion of the contributions from the gamma gluon fusion processes can considerably enlarge the power of the scaled factorial moments. The possibility for probing the anomalous scaling behaviors of probability moments of the transverse energies in HERA calorimeter environment is also discussed.
\end{abstract}


Recent HERA-experiments on deep-inelastic electron-proton (ep) scattering (DIS) in the low $x_{B}$ kinematic range have clearly shown the existence of a distinct class of events. These events are characterized by the fact that there is no hadronic energy flow in a considerably large interval of pseudo-rapidity $\eta$ adjacent to the proton beam direction. Our present understanding of DIS could be inadequate at low- $x_{B}$ because additions to the leading order QCD-based partonic picture are likely to be substantial. A natural interpretation of these so called "large rapidity gap" events is based on the hypothesis that the deep-inelastic scattering process involves the interaction of the virtual boson probe with a colorless component of the proton. Hence there is no chromodynamic radiation in final state immediately adjacent to the direction of the scattered proton or any proton remnant. What is this colorless component originating from the nucleon? The large rapidity gap events discovered in deep inelastic scattering at HERA目 are usually interpreted in terms of pomeron-exchanged modell. Although this seems to work reasonably well phenomenologically, there is yet no satisfactory understanding of the pomeron structure and its interactions mechanism. In this respect, it is helpful to probe the properties of such objects in the non-traditional aspects, in addition to the traditional measures such as rapidity gap distribution, structure function and the averaged cross section and so ont. In the present note we wish to point out that useful information about the exchanged colorless object in diffractive lepton-nucleon scattering process can be extracted by studying scaling behavior and fractality (intermittency) of the final state of the colorless component in proton excited by the virtual boson probe.

The manifestation of fractality (intermittency) in high energy multiparticle production process is the anomalous scaling

$F_{q}(\delta x)=F_{q}(\Delta x)\left(\frac{\Delta x}{\delta x}\right)^{\phi_{q}} \quad$ as $\quad M \rightarrow \infty, \quad \delta x \rightarrow 0$

of $q$-order factorial moments (FM's) $F_{q}$, defined as

$F_{q}(\delta x)=\frac{1}{M} \sum_{m=1}^{M} \frac{\left\langle n_{m}\left(n_{m}-1\right) \ldots\left(n_{m}-q+1\right)\right\rangle}{\left\langle n_{m}\right\rangle^{q}}$,

where $x$ is some phase space variable, e.g. (pseudo-)rapidity, the scale $\delta x=\Delta x / M$ is the bin width for a $M$-partition of the region $\Delta x$ in consideration, $n_{m}$ is the multiplicity in the 
$m$ th bin. Since the factorial moments can remove the statistic noise around the probability and associated directly with the scaled moments of probabilities, the scaling exponent $\phi_{q}$ in Eq.( 1), called intermittency index, characterizing the strength of dynamical fluctuation, is connected $\mathbf{1}$ with the anomalous fractal dimension $d_{q}$ of rank $q$ of the spatial-temporal evolution of high energy collisions, $d_{q}=\phi_{q} /(q-1)$. A possible cause leading to the powerlaw of FM's of final particles in high-energy collisions is that the emitting source of final hadrons is self-similar fractall . Furthermore, DIS experiments and the empirical analysest. show that the gluon-density in the nucleon in the low- $x_{B}$ kinematical region is much higher than those for quarks/antiquarks, and it is increasing with decreasing $x_{B}$ l. In this soft gluon system where the gluons interact with each other in complicated dynamic processes, it has been proposed 8 that the gluons may 'self-organize' into the clusters in the dissipative gluon system by self-organized criticality (SOC) 1 , and the colorless component in proton can be regarded as color singlet gluon cluster 10 . The spatial-temporal structure of the SOC-cluster (or BTW-cluster) is self-similar fractal 1 . So it is feasible to study the fractal structure of the colorless component of the proton by measuring the anomalous scaling behaviors of factorial moments of the final state particles originating from the scattering of virtual photon and colorless object in the diffractive lepton-nucleon scattering.

The $x_{B}$-dependence of scaling behaviors of FM's, and in particular that of intermittency index $\phi_{q}\left(Q^{2}, x_{B} ; x_{\mathbb{P}}\right)$ for fixed $Q^{2}$ (and $\left.x_{\mathbb{P}}\right)$, plays a distinguished role in such studies. This is because, when the virtual photon $\gamma^{*}$ originating from the incident lepton is absorbed by the nucleon, its energy-momentum $q \equiv\left(q^{0}, \vec{q}\right)$ is in fact absorbed by a virtual colorless component of the nucleon in the lepton-nucleon diffractive scattering. In a fast moving reference frame, for example the lepton-nucleon center-of-mass frame, where the nucleon's momentum $\vec{P}$ is large in high-energy collisions, the time interval $\tau_{\text {int }}$ in which the absorption process takes place (it is known as the lepton-nucleon interaction/collision time) can be estimated by making use of the uncertainty principle. In fact, by calculating $1 / q^{0}$ in this reference frame we obtain 1 : 
$\tau_{\text {int }} \sim \frac{4|\vec{P}|}{Q^{2}} \frac{x_{B}}{1-x_{B}}$.

This means, for given $\vec{P}$ and $Q^{2} \equiv-q^{2}$, the interaction time $\tau_{\text {int }}$ is directly proportional to $x_{B}$ in the low- $x_{B}\left(x_{B} \ll 1\right)$ kinematic range. In other words, $x_{B}$ is a measure of the time-interval in which the absorption of $\gamma^{*}$ by the space-like virtual colorless object takes place. Hence, by studying the $x_{B}$-dependence of the intermittency index $\phi_{q}\left(Q^{2}, x_{B} ; x_{\mathbb{P}}\right)$, we are not merely probing the anomalous scaling behaviors of the collision process between $\gamma^{*}$ and the colorless object which we hereafter call $c_{0}^{*}$. Since this hadronization process of the virtual colorless object $c_{0}^{*}$ is initiated by the interaction with $\gamma^{*}$, we are also examing whether/how the hadronization process changes with the interaction time $\tau_{\text {int }}$. This question is of considerable interest, because a virtual photon $\gamma^{*}$ can (logically) only be absorbed by virtual systems $\left(c_{0}^{*} s\right)$ whose lifetimes $\left(\tau_{c}^{*}\right.$ s) $\left.s\right)$ are longer than interactive time $\tau_{\text {int }}$ (i.e. $\left.\tau_{\text {int }} \leq \tau_{c}\right)$. That is, the average lifetime $\left\langle\tau_{c}\right\rangle$ of the $c_{0}^{*}$ s, which can absorb a $\gamma^{*}$ associated with interaction-time $\tau_{\text {int }}$, is a function of $\tau_{\text {int }}$. Hence, from the $x_{B}$-dependence of the scaling behavior of FM's, in particular from that of the corresponding $\phi_{q}\left(x_{B}, Q^{2} ; x_{\mathbb{P}}\right)$ 's, we can in principle find out whether/how the dynamic fluctuation and the fractal structure in the spatial-temporal evolution originating from the exchanged colorless object $c_{0}^{*}$ depends on its average lifetime $\left\langle\tau_{c}\right\rangle$ of the $c_{0}^{*}$ s.

The $Q^{2}$-dependence of the scaling behavior of the FM's is also of considerable interest. This is because, in photon-proton scattering experiments, not only those with real $\left(Q^{2}=\right.$ $0)$ photons but also those with space-like $\left(Q^{2}>0\right)$ photons where $Q^{2}$ is not too large $\left(\leq 1 \mathrm{GeV}^{2} / c^{2}\right.$, say) have very much in common with hadron-hadron collisions. Having in mind that the index of intermittency for hadron-hadron scattering is smaller than that for electron-positron annihilation processes $₫$, we are led to the following questions: Do we expect to see a stronger $Q^{2}$-dependence when we increase $Q^{2}$ from zero to 10 or $100 \mathrm{GeV}^{2} / \mathrm{c}^{2}$, say? Is this also a way to see whether space-like photons at large $Q^{2}$ "behave like hadrons" in such interactions?

While waiting for data to perform the above-mentioned analysis, let us now consider the 
following two phenomenological models as illustrative examples:

(A). If the colorless object $\left(c_{0}^{*}\right)$ is a quark-antiquark pair (formed by interacting gluons) which exists in the time-interval when the virtual photon $\gamma^{*}$ is absorbed by the object, we shall see the following: Especially when $Q^{2}$ is sufficiently large, the incoming $\gamma^{*}$ (the transverse dimension is expected to be proportional to $\left.1 / Q^{2}\right)$ will hit the quark $(q)$ or the antiquark $(\bar{q})$ and make them fly apart symmetrically with respect to the center of mass the $\gamma^{*} q \bar{q}$ system - similar to the $q \bar{q}$-pair produced in $e^{+} e^{-}$-collisions (with respect to the center of mass the $q \bar{q}$ system). That is, in this case, the final-state-hadrons of an event are fragmentation-products of the quark and/or the antiquark, and hence they are expected to show characteristic features similar as those observed in the reaction $e^{+} e^{-} \rightarrow$ hadrons at the same c.m.s. energy. It is interesting to see that the very recent inclusive measurements performed at HERAl in diffractive electron-proton scattering show the following: Both the scaled longitudinal momentum $\left(x_{F}\right)$ distribution and the transverse momentum $\left(p_{\perp}\right)$ distribution are strikingly symmetric with respect to the center-of-mass of the photon and the struck colorless object; and the general features of these distributions are very much the same as those observed in electron-position collision processes. These facts strongly suggest that a more detailed comparison between these two collision processes would be useful.

For this purpose, we made use of the Monte Carlo (MC) program JETSET 13 . We generated 50,000 MC events, and calculated the second normalized factorial moment in 3dimensional $\left(\eta, p_{\perp}, \phi\right)$ phase space at the given $q \bar{q}$-c.m.s energy $\sqrt{s}$, where the pseudorapidity $\eta$, transverse momentum $p_{\perp}$ and the azimuthal angle $\phi$ are defined with respect to the sphericity axis of the event. The usual cumulative variables $X$ translated from $x=\left(\eta, p_{\perp}, \phi\right)$, i.e.12

$X(x)=\int_{x_{\min }}^{x} \rho(x) d x / \int_{x_{\min }}^{x_{\max }} \rho(x) d x$

were used to rule out the enhancement of $F_{q}$ from a non-uniform inclusive spectrum $\rho(x)$ of the final hadrons. The obtained results have been collected in Fig.1a in double logarithmic $F_{2}$ vs $\mathrm{M}$ plots for different $\sqrt{s}$ (or $M_{X}$, which is the invariant mass of the $\gamma^{*} c_{0}^{*}$ system 
in a corresponding diffractive lepton-nucleon scattering event). It is clear that the higher the invariant mass of $\gamma^{*} c_{0}^{*}$ is, the larger the power of FM's, i.e. the stronger the dynamic fluctuation is. In the very low invariant mass $(\sqrt{s}=4.5 \mathrm{GeV}$, say), the powers of FM's become less than 0 , which can be referred to the constraint of the momentum conservation in the hadronization process 14 .

(B). Based on pomeron-exchanged model目, several Monte Carlo generators, such as POMPYT司, RAPGAP 16 have been set up. These models have been used to describe quite well the HERA data in the global and averaged features, such as the rapidity gap distribution, the diffraction and total cross section and so on 1 . The theoretic models are usually confronted with incorrigible discrepancy, when the data about the locally nonstatistic fluctuation in small phase space are involved in the comparison with themt. No evidence has shown that this kind of locally dynamic fluctuation could be certainly referred only to the hadronization process but have nothing to do with the initial stage of high energy collisions. In this respect, it is also relevant to see whether the features of scale invariance and fractality in small phase space of the lepton-nucleon diffractive processes, specially their dependence upon the diffractive variables can be compatible with the pomeron type of model. In the following we take RAPGAP as an example, in which the virtual photon $\left(\gamma^{*}\right)$ interacts directly with a parton constituent of the pomeron either in lowest order (Fig.2a) or in $O\left(\alpha_{\mathrm{em}} \alpha_{s}\right)$ order — via the photon-gluon fusion (Fig.2b). In both cases a color octet remnant is left at low $p_{T}$ with respect to the pomeron and hence also to the proton. The higher order gluon emission is simulated with the Color Dipole Model (ARIADNE) 17 and the hadronisation is performed using the JETSET13. The pomeron flux factor $f_{P \mathbb{P}}\left(t, x_{\mathbb{P}}\right)$ and pomeron structure function $G(\beta)$ are taken as

$f_{P \mathbb{P}}\left(t, x_{\mathbb{P}}\right)=\frac{\beta_{P \mathbb{P}}^{2}(t)}{16 \pi} \beta^{1-2 \alpha_{\mathbb{P}}(t)}$,

given by Berger et al. and Streng 2 , and

$$
\beta G(\beta)=6(1-\beta)^{5},
$$


when the pomeron is made of 2 'unrealistically hard' gluons as suggested by Ingelman and Schlein2. Here the kinematic variables $t, x_{\mathbb{P}}$ and $\beta$ are defined as $t=\left(P-P^{\prime}\right)^{2}$, $x_{\mathbb{P}}=\frac{q \cdot\left(P-P^{\prime}\right)}{q \cdot P}$, and $\beta=\frac{-q^{2}}{2 q \cdot\left(P-P^{\prime}\right)}$, where $P^{\prime}$ is the 4-momentum of the final state colorless remnant of nucleon. The pomeron Regge trajectory is given by $\alpha_{\mathbb{P}}(t)=1+\epsilon+\alpha^{\prime} t, \epsilon \approx 0.085$ and the slope $\alpha^{\prime}=0.25$ obtained from a fit to datal.

We generated 500,000 RAPGAP events, and divided the whole sample into 10 subsamples according to the invariable mass $M_{X}$ of $\gamma^{*} c_{0}^{*}$ in the MC event. The scaling behaviors of FM's for different $M_{X}$ intervals are shown in Fig. 1b. The dependence of scaling behaviors of FM's upon $M_{X}$ is similar with the result of JETSET in Fig.1a, i.e. the powers increase for increasing scattering energy of $\gamma^{*} c_{0}^{*}$. But it is noticeable that the intermittency index $\phi_{2}$ for a given $M_{X}$ interval is much larger in RAPGAP than that in JETSET. Having in mind that in Fig. 1a the colorless object is considered as a quark-antiquark pair formed by gluons and the Feymann graph of the diffractive process in this aspect is just same as shown in Fig. 2a, the difference between cases (A) and (B) is that the higher-order photon-parton interaction has been taken into account in RAPGAP (see Fig. 2b). It is understandable since the branch number of the parton cascading process in parton shower level is larger when the gamma gluon fusion is involved in Fig. 2b, while it is believed generally that the color-string type of models are referred in large part to the randomly cascading process of parton energy in perturbative phase, so the fractal in the evolution processes with longer cascade branch would be stronger.

In order to see the dependence of dynamic fluctuation upon the other diffractive variables as we argued above, we divided the Monte Carlo sample into 10 subsamples according to $x_{B}$ and $Q^{2}$. The results of scaling behaviors of FM's are shown in Figs. 3a and b respectively. This example explicitly shows how the proposed method can be used to analyze the feature of the evolution precess of the colorless object in diffractive lepton-nucleon scattering processes. The significant $x_{B}$-dependence of the intermittency index observed in this example not only shows that the hadronic final states depend very much on the average lifetime of the exchanged colorless object $c_{0}^{*}$ if $c_{0}^{*}$ can be indeed considered as a pomeron as simulated in 
RAPGAP, but also the following: The longer the average lifetime of $c_{0}^{*}$ is, the stronger the fractal structure of the space-time evolution processes originating from the $\gamma^{*} c_{0}^{*}$ 's interaction will be. The smaller the $Q^{2}$ becomes, i.e. the larger transverse dimension of virtual $\gamma^{*}$ is, the more $\gamma^{*}$ "behaves like a hadron".

Since $x_{\mathbb{P}}$ may be interpreted as the fraction of the 4-momentum of the proton carried by the exchanged colorless objects, and $\beta$ as the fraction of the 4-momenta of the exchanged objects carried by the parton interacting with the virtual boson, it meaningful to calculate the dependence of scaling behavior upon $x_{\mathbb{P}}$ and $\beta$ (Fig. $3 \mathrm{c}$ and $\mathrm{d}$ respectively). It seems from RAPGAP that only when the momentum of colorless object is large enough, can scaling behavior of the final state originating from $\gamma^{*} c_{0}^{*}$ be significant; and it is clear from Fig. 3d that the dynamic fluctuation in the diffractive scattering don't increase monotonously for increasing the fraction of energy of stricken parton in the pomeron.

Last but not least, the following should be mentioned. Having in mind that jets have been observed (see e.g. the second paper in Ref. and the papers cited there.) in diffractive electron-proton scattering processes, and HERA calorimeters have been used to measure transverse energies distribution of the collision events to study jet structure, it is meaningful to measure the scaling behavior of evolution process of $\gamma^{*} c_{0}^{*}$ by using the distribution of transverse-energies in phase space, instead of conventional multiplicity analysis. Here, the transverse-energy $E_{\perp}$ is measured on an event-by-event bases with respect to the axis of the virtual photon. According to the most recent experimental knowledget we expect to see that the distributions of $E_{\perp}$ in phase space in such collision events are symmetric with respect to this axis, and symmetric with respect to the origion of the c.m.s. frame of the colliding objects $\gamma^{*} c_{0}^{*}$. As is known 3 , the factorial moments defined as Eq.( (2), of multiplicity of final state particles can rule out the statistical fluctuation around probability $p_{m}$ by which a particles appear in the $m^{\prime}$ th bin of phase space, i.e. $F_{q}=C_{q} \equiv(1 / M) \sum_{m=1}^{M}\left\langle p_{m}^{q}\right\rangle /\left\langle p_{m}\right\rangle^{q}$. In order to measure the scaling behavior of probability moments of transverse energies, a straightforward manner following the usual procedure is to introduce an energy-unit $\varepsilon$ and 
write the 'transverse energy factorial moment' $F_{q}^{(E)}$ as $\left\langle E_{\perp}\left(E_{\perp}-\varepsilon\right) \cdots\left[E_{\perp}-(q-1) \varepsilon\right]\right\rangle /\left\langle E_{\perp}\right\rangle^{q}$. It is clear that $E_{\perp} / \varepsilon$ can be considered as integers, provided that $\varepsilon$ is sufficiently small. Under this condition, the statistical fluctuations around transverse energies can be removed in $F_{q}^{(E)}$ in the same way as that in $F_{q}$ defined in Eq.( 2). But, this means, there is a dependence on an arbitrary parameter $\varepsilon$, when we use $F_{q}^{(E)}$ ! In order to check the possibility of getting rid of this kind of arbitrariness in the practice, let's introduce a variable, $\lambda \equiv \varepsilon / E_{\perp}^{t}$, i.e. the ratio between the arbitrarily chosen energy-unit $\varepsilon$ and total transverse $E_{\perp}^{t}$ of an event. We generate the transverse energies of the 'events' in the phase space by computer according to the Bernoulli distribution of $\lambda$. It is obvious that the slope in the double logarithmic $F_{q}^{(E)}$ vs M plot has to be flat, since there is no dynamical fluctuation in this sample. In this sample, we calculated the transverse energy moment $R_{q}^{(E)} \equiv\left\langle E_{\perp}^{q}\right\rangle /\left\langle E_{\perp}\right\rangle^{q}$ for different choices of $\lambda$. The corresponding $R_{2}^{(E)}$ vs M plots is shown in Fig.2. Here we see that the transverse energy moment $R_{2}^{(E)}$ can be considered as a good approximation for $F_{2}^{(E)}$, i.e. probability moments of transverse energies, when $\varepsilon$, which depends upon the resolving power of the calorimeters, is of the order of $10^{-3}$ of the total $E_{\perp}$ in the events under consideration.

In conclusion, we have shown in this note that the colorless object $c_{0}^{*}$ in diffractive leptonnucleon scattering can be probed in a model-independent way, i.e. by performing the scaled factorial moment analyses for the hadronic final states originating from the scattering of virtual boson $\gamma^{*}$ and the $c_{0}^{*}$ on an event-by-event bases. The JETSET and RAPGAP has been discussed as illustrative examples. It is shown in particular that higher-order interactions in hard parton level are important in the intermittency analysis of the diffractive scattering, Furthermore, we also pointed out that HERA calorimeter environment is a possible place to carry out transverse-energy moment analyses of intermittency for probing dynamical fluctuations.

Most of the ideas discussed in this paper were generated in conversations with Meng Ta-chung to whom I am grateful for patience and understanding. Thanks are also due to C. Boros, D. H. E. Gross, Z. Liang, R. Rittel, K. D. Schotte and K. Tabelow for helpful 
discussions, H. Jung for the patient helps in RAPGAP Monte Carlo generator. I also thank Alexander von Humboldt Stiftung for the financial support. 


\section{REFERENCES}

1. M. Derrick et al. ZEUS Collaboration, Phys. Lett. B315, 481(1993); Phys. Lett. B332, 228(1994); T. Ahmed et al. H1 Collaboration, Nucl. Phys. B429, 477(1994); Phys. Lett. B348, 681(1995); For a recent review, see e.g. N. Cartiglia, Diffraction at HERA, hep-ph/9703245; and the papers cited therein.

2. G. Ingelman and P. Schlein, Phys. Lett. B152, 256(1985); E. Berger, J. Collins, D. Soper and G. Sterman, Nucl. Phys. B286, 704(1987); A. Donnachie and P. Landshoff, Phys. Lett. B191, 309(1987); K. H. Streng, in: Proc. of the Workshop Physics at HERA, Hamburg (1987), ed. R. D. Peccei; and the papers cited therein.

3. A. Bialas and K. Peschanski, Nucl. Phys. B273(1986)703; B308(1988)857.

4. For a recent review, see e.g., N. Schmitz, in: Proc. XXI Int. Symp. on Multiparticle Dynamics, Wuhan, 1991, eds. Y. Wu and L. Liu (World Scientific, Singapore, 1992) P.377; E.A. De Wolf, I.M. Dremin and W. Kittel, Phys. Rep. 270, 1(1996).

5. P. Lipa and B. Buschbeck, Phys. Lett. B223(1989)465; R. C. Hwa, Phys. Rev. D41(1990)1456; Liu Lianshou, Zhang Yang and Wu Yuanfang, Z. Phys. C69(1996)323; Zhang Yang, Liu Lianshou and Wu Yuanfang, Z. Phys, C71(1996)499.

6. A. Bialas, Nucl. Phys. A545(1992)285c; Acta Phys. Pol. B23(1992)561.

7. See e.g. S. Aid et al. (H1 Collaboration), Phys. Lett. B354, 494(1995) and M. Derrick et al. (ZEUS Collaboration), Phys. Lett. B345, 576(1995).

8. C. Boros, T. Meng, R. Rittel and Y. Zhang, hep-ph/9704285.

9. P. Bak, C. Tang and K. Wiesenfeld, Phys. Rev. Lett. 59, 381(1987); Phys. Rev. A38, 364(1988). For a recent review, see P. Bak, How nature works(Springer-Verlag, New York, 1996).

10. F. E. Low, Phys. Rev. D12, 163(1975); S. Nussinov, Phys. Rev. Lett. 34, 1286(1975) 
and Phys. Rev. D14, 246(1976); C. Boros, Liang Zuo-tang and Meng Ta-chung, Phys. Rev. D54, 6658(1996).

11. The lifetime $\tau_{\text {int }}$ of virtual photon in the lepton-nucleon center-of-mass frame in DIS can be easily formed in the following simple kinematic exercise: $k^{\prime 2} \equiv m_{e}^{2}=(k-q)^{2}=$ $m_{e}^{2}+q^{2}-2 k \cdot q$, where $k \equiv\left(k^{0}, \vec{k}\right), k^{\prime} \equiv\left(k^{\prime 0}, \vec{k}^{\prime}\right), q \equiv\left(q^{0}, \vec{q}\right)$ and $P \equiv\left(P^{0}, \vec{P}\right)$ are the 4-momentum of incident lepton, outgoing lepton, exchanged virtual photon and incident nucleon in lepton-nucleon center-of-mass frame respectively, $m_{e}$ and $m_{p}$ are the invariance mass of lepton and nucleon. Taking into account $\vec{k}=-\vec{P}$ and $|\vec{k}| \gg$ $m_{e},|\vec{P}| \gg m_{p}$, then $m_{e}^{2} \simeq m_{e}^{2}+q^{2}-2|\vec{P}| q^{0}-2 \vec{P} \cdot \vec{q}=m_{e}^{2}+q^{2}-2|\vec{P}| q^{0}-2 q^{0} \sqrt{|\vec{P}|^{2}+m_{p}^{2}}+$ $2 P \cdot q$, i.e. $q^{2}=\frac{2 P \cdot q+q^{2}}{2 \sqrt{|\vec{P}|^{2}+m_{p}^{2}}+2|\vec{P}|} \simeq \frac{2 P \cdot q-Q^{2}}{4|\vec{P}|}$. So the lifetime of virtual photon is $\tau_{\text {int }} \sim \frac{1}{q^{0}} \sim$ $\frac{x_{B}}{1-x_{B}} \frac{4|\vec{P}|}{Q^{2}}$.

12. A. Bialas and M. Gazdzicki, Phys. Lett. B252, 483(1990); W. Ochs, Z. Phys. C50, $339(1991)$.

13. T. Sjostrand, Comput. Phys. Commun. 39, 347(1986).

14. Liu Lianshou, Zhang Yang and Deng Yue, Z. Phys. C73(1997)535.

15. P. Bruni and G. Ingelman, DESY-93-187, Proc. Rurophysics Conf. on HEP, Marseilles 1993, Eds. J. Carr and M. Perottet, P.595.

16. H. Jung, Comput. Phys. Commun. 86(1995)147.

17. L. Lönnblad, Comput. Phys. Commun. 71(1992)15. 


\section{FIGURES}

Fig. 1. The scaled factorial moments $F_{2}$ versus the number $M$ of subintervals of 3 -dimensional $\left(\eta, p_{\perp}, \phi\right)$ phase space in log-log plot, and the second-order intermittency index $\phi_{2}$ in corresponding sample set. (a). The MC result of JETSET 7.4 in different cms energy $\sqrt{s} .50000$ events are generated in each sample set; (b). That of RAPGAP in corresponding invariable mass $M_{X}$ interval with $N_{\text {event }}$ events in each subsample. The pomeron flux and pomeron structure function has given by Eqs. (5) and ( 6) in the text as a default of the MC generator.

Fig. 2. The basic processes included in the RAPGAP16 implementation for inelastic lepton scattering on a pomeron: (a) the lowest order process for hard parton level; (b) the $O\left(\alpha_{\mathrm{em}} \alpha_{s}\right)$ order process for gamma gluon fusion.

Fig. 3. The dependence of 2-order intermittency index $\phi_{2}$ in RAPGAP 16 Monte Carlo implementation upon diffractive variables: (a) $x_{B}$, (b) $Q^{2}$, (c) $x_{\mathbb{P}}$ and (d) $\beta$.

Fig. 4. The 2-order transverse energy moment $R_{2}^{(E)}=\left\langle E_{\perp}^{2}\right\rangle /\left\langle E_{\perp}\right\rangle^{2}$ as functions of partition number of phase space $M=\Delta / \delta$ in log-log plot, when the transverse energy $E_{\perp}$ in units of $\varepsilon$ in subinterval $\delta$ is stochastically produced according to Bernoulli distribution. Here, $\lambda=\varepsilon / E_{\perp}^{t}$, and $E_{\perp}^{t}$ is the total transverse energy in the considered phase space $\Delta$. 


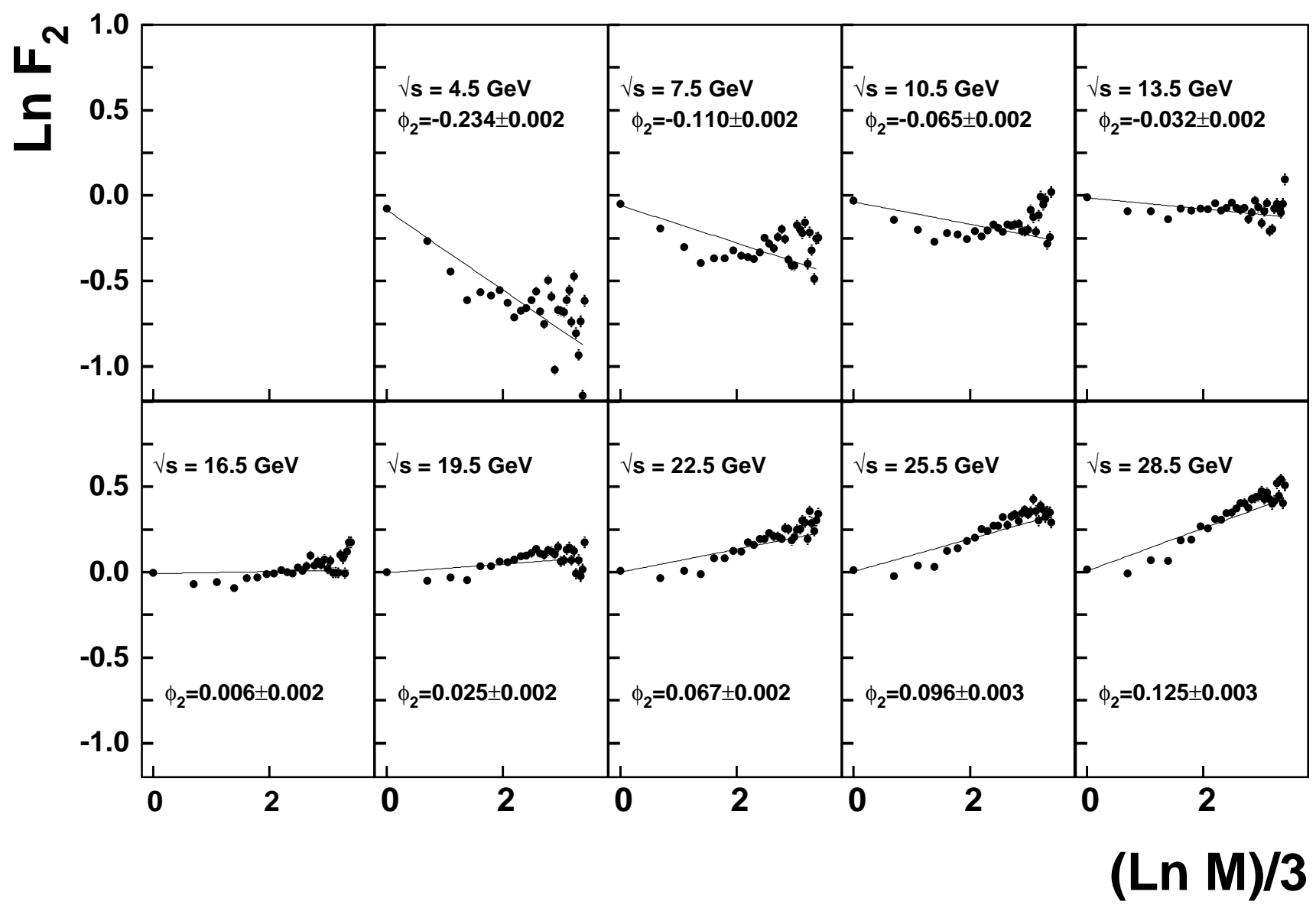

Fig. 1a 


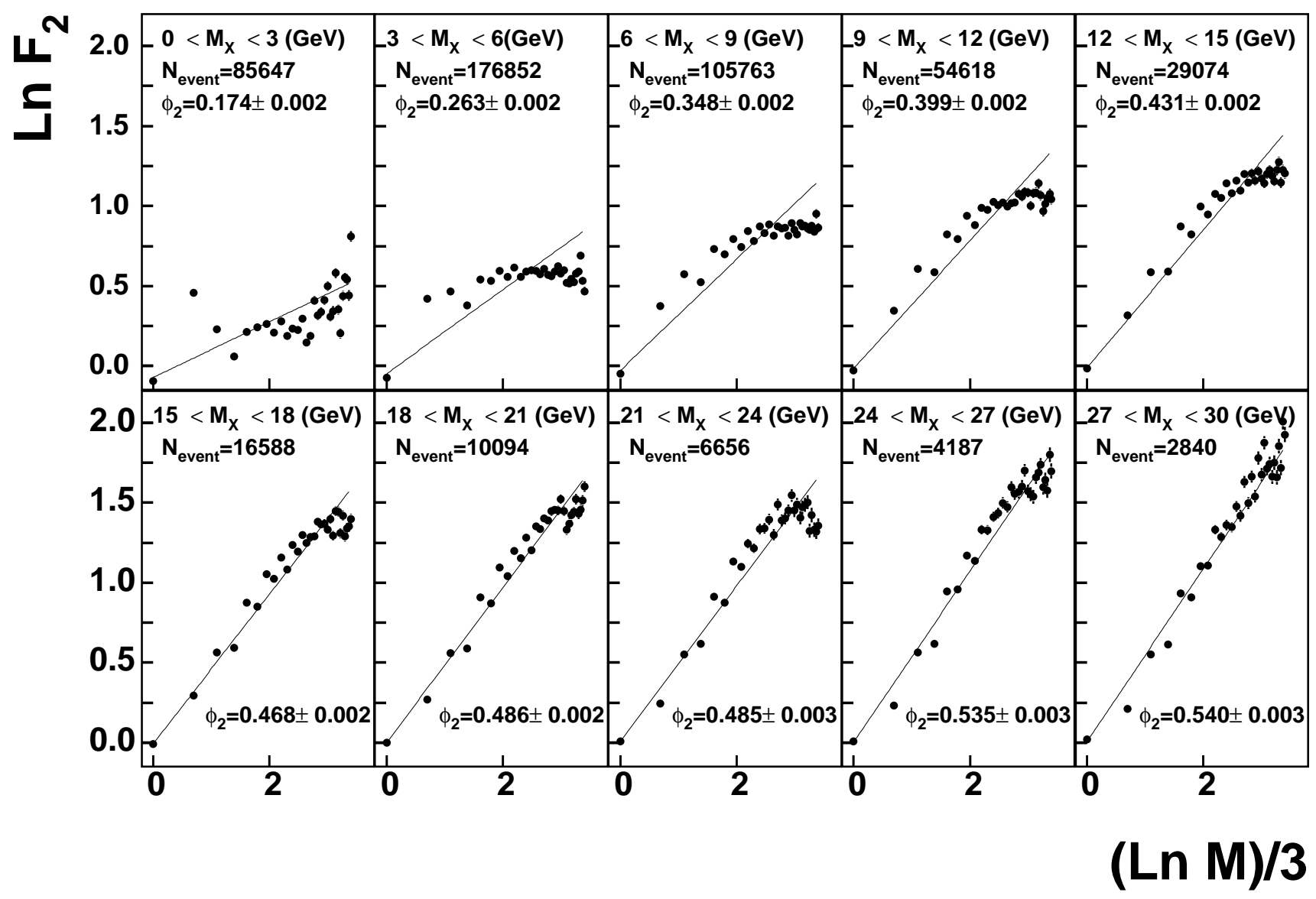

Fig. 1b 


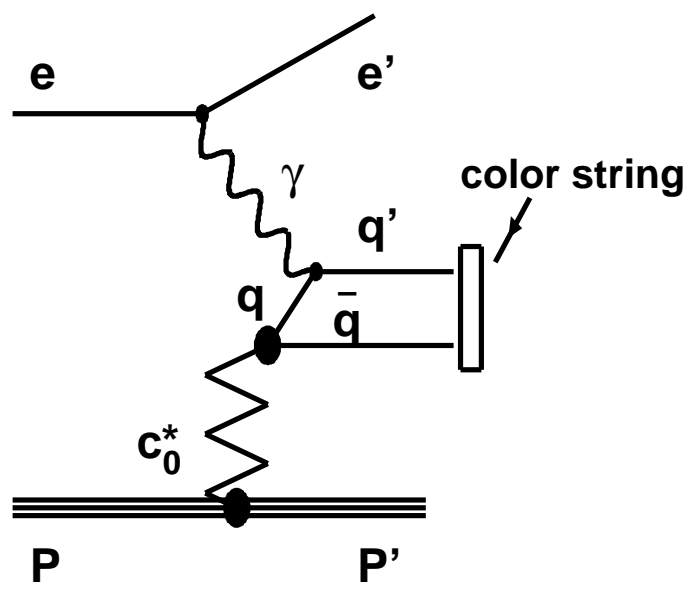

(a)

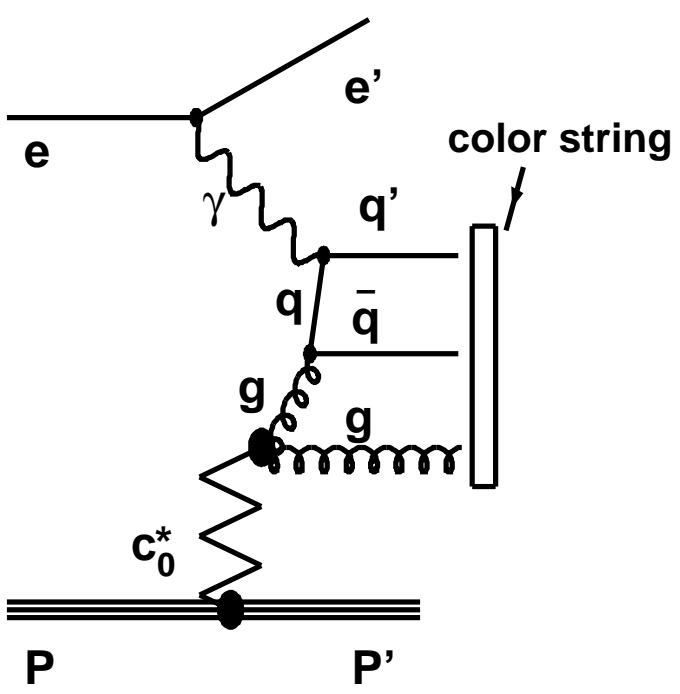

(b)

Fig. 2 

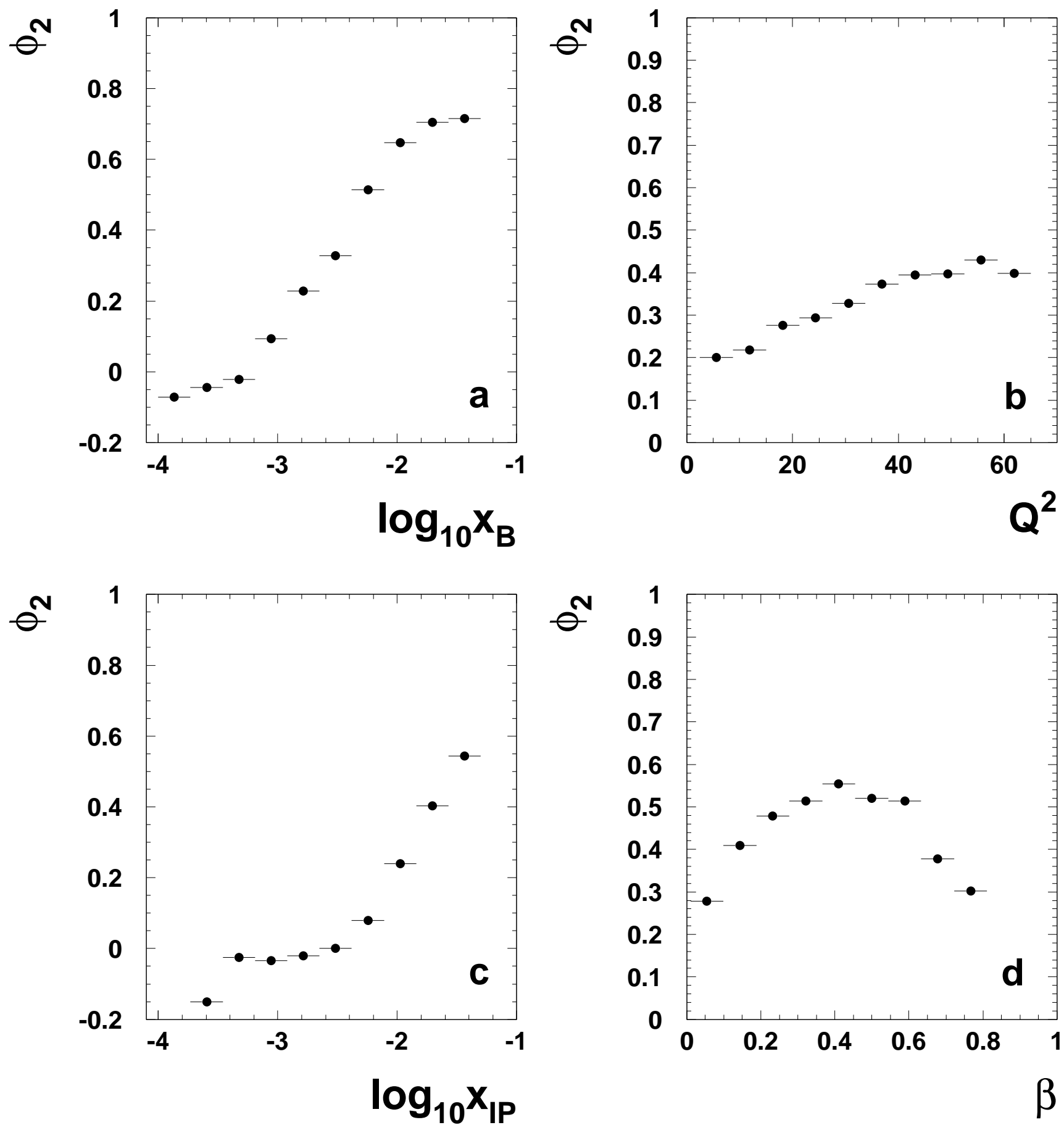

Fig. 3 


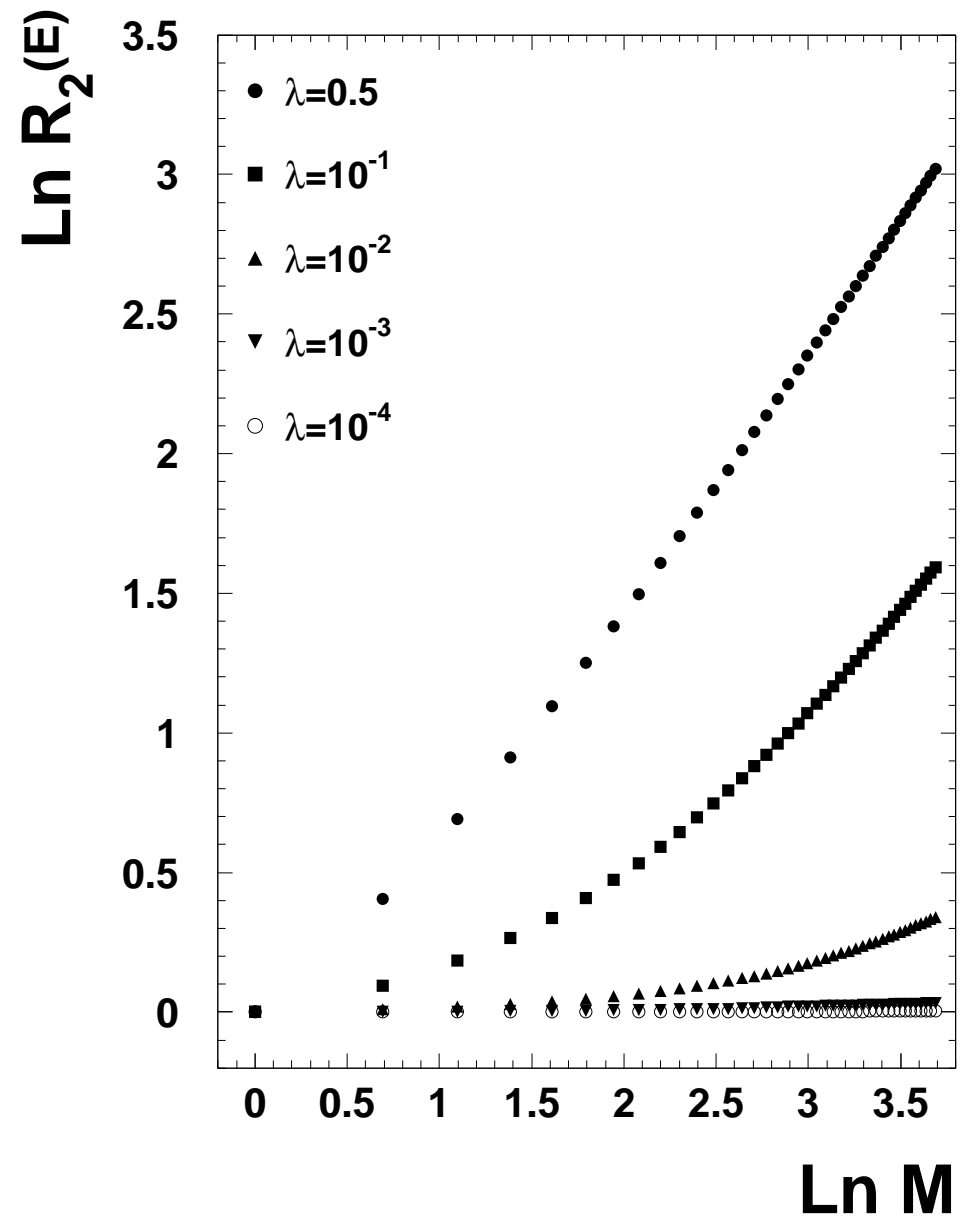

Fig. 4 